\title{
Spectroscopy in Utrecht: A Brief History
}

\author{
Frank Verbunt · Johan Bleeker
}

Received: 30 September 2010 / Accepted: 4 November 2010

(C) The Author(s) 2010. This article is published with open access at Springerlink.com

\begin{abstract}
In the first half of the 20th century, Leonard Ornstein turned the Physics Laboratory of Utrecht University into a center for testing the nascent quantum mechanics through accurate measurements of line intensities in flame spectra. The microphotometer of instrument builder Willem Moll was the key to this success. Marcel Minneart used this instrument to study stellar spectra at the Utrecht Astronomical Institute, and in particular for the Photometric Atlas of the Solar Spectrum. When the opportunity came in the 1960s to build instruments for research from space, it was natural that Kees de Jager opted for spectroscopy, in X-ray observations of the Sun. The expertise developed in this program allowed the Space Research Organisation of the Netherlands to be a principal player in the development of $\mathrm{X}$-ray spectrographs on the Einstein, EXOSAT, Chandra and XMM-Newton satellites.
\end{abstract}

Keywords History of physics · History of astronomy · Instrumentation: spectrographs

\section{Introduction}

At the start of a meeting on high-resolution X-ray spectroscopy in Utrecht, it is perhaps not inappropriate to have a brief look at the history of spectroscopy in Utrecht. The first part of this paper is about optical spectroscopy in the Utrecht physics department from the end of the 19th century to the period in the 1930s when the work by Ornstein and his group had a pronounced impact on the development of quantum mechanics. Our discussion of this period is based mainly on the PhD thesis (in Dutch) by Heijmans (1994). The second part describes the continuation of this work at the Astronomical Institute, and is based mainly on

F. Verbunt $(\bowtie) \cdot$ J. Bleeker

Astronomical Institute Utrecht University, Utrecht, The Netherlands

e-mail: f.w.m.verbunt@uu.nl

J. Bleeker

e-mail: J.A.M.Bleeker@sron.nl

F. Verbunt $\cdot$ J. Bleeker

SRON Netherlands Institute for Space Research, Utrecht, The Netherlands

Published online: 31 December 2010 
a biography (also in Dutch) of Minnaert, by Molenaar (2003). The final part describes the change from optical to X-ray spectroscopy at the Utrecht branch of the Netherlands Institute for Space Research.

\section{Quantitative Spectroscopy for Quantum Mechanics}

\subsection{Origins: Buys Ballot and Julius}

In 1863 the Dutch government passed a law on the education system which was to have a deep and lasting impact on the study of the natural sciences in the Netherlands, by establishing a new type of secondary education: the Hogere Burger School (higher school for citizens), the HBS. The HBS emphasized education in the natural sciences, and provided the universities with students from middle and lower income groups who in earlier times would not have had higher education. The HBS directly lead to a flowering of the natural sciences in the Netherlands, as witnessed by a number of Dutch Nobel-Prize laureates in physics and chemistry in the early 20th century (Willink 1998).

The first university students who arrived from the HBS discovered to their surprise that the experimental labs at the universities were outdated with respect to the high-school laboratories, and in Utrecht Buys Ballot, who in 1853 had founded the Sonnenborgh Observatory for meteorology and astronomy, founded a new physics laboratory, devoted mainly to teaching, in 1877.

As an intermezzo we may mention here the influence that Buys Ballot had on the career of a certain Wilhelm Conrad Röntgen, a youngster who refused to tell on prank of a fellow student at the Lower Technical School, and as a result was thrown out. Thus without a diploma, he would not be able to obtain a degree at Utrecht University when he arrived there in 1865. Buys Ballot was impressed sufficiently with the qualities of his pupil to write a recommendation letter for him to the ETH in Zürich, Switzerland, and so set Röntgen on track for his career.

In 1888 Buys Ballot asked W.H. Julius, one of his students in the new laboratory, to study the heat radiation of solids, in particular the spectral energy distribution, following in the footsteps of Kirchhoff and Wien. Hoping to explain the spectra of solids by the emission properties of their basic building blocks, Julius also measured flame spectra of molecules. In 1896 Julius became the director of the physics laboratory and shifted his interest to radiation of the Sun. In these pre-quantum-mechanics days he devised a theory to explain the Fraunhofer lines in the solar spectrum with anomalous dispersion, and to measure these lines he built a heliostat spectrograph on the roof of the laboratory. Together with astronomers Nijland and Van der Bilt of the Astronomical Observatory in Utrecht, Julius also organized eclipse expeditions, for example to Maastricht in 1912, to measure chromospheric lines.

\subsection{The Microphotometer and Quantum Numbers}

Most measurements of the heat radiation were made with thermocouples. The brilliant instrument builder Willem Jan Henry Moll in the course of many years perfected the measuring apparatus into Moll's microphotometer, as it became known, the work horse of the spectroscopic work done in Utrecht in the 1920s and beyond (van Cittert 1926; Moll 1921, 1926). Moll started by improving the thermocouples by better insulation and by better selection of metals. He then attached a mirror to the galvanometer and used a beam of light reflecting on it to magnify the excursion and register it on a slowly moving photographic plate, thus achieving automatic registration. 
Fig. 1 Moll's microphotometer in 1919. For explanation see text

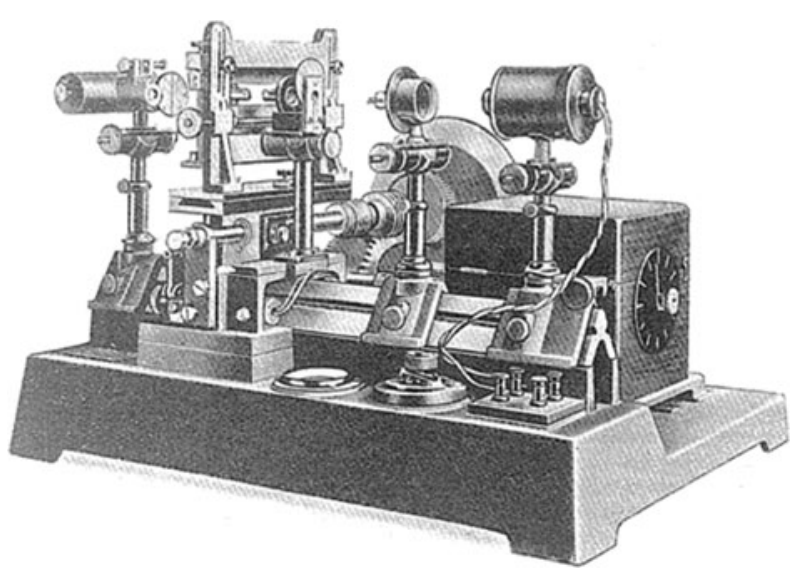

Figure 1 shows Moll's microphotometer as it was in 1919. The light beam passes from its source on the right via a lens through the photographic plate on which the spectrum is recorded onto the thermocouple on the left. This photographic plate is mounted in a frame, and the motion of the frame is mechanically coupled to the motion of a new photographic plate that records the beam reflected from the mirror on the thermocouple. Thus the spectrum could be scanned automatically, as the density (i.e. measure of darkness) on the photographic plate with the spectrum is converted into a tracing (excursion) on the new photographic plate.

Julius was succeeded as director of the physics laboratory in 1918 by Leonard Salomon Ornstein. Although trained as a theoretical physicist, he turned the Utrecht institute into a mainly experimental laboratory. He contacted industry to align the experimental physics with its needs, thereby enhancing the level of both industry and the physics laboratory. (Some 20 of Ornsteins 94 (!) graduate students would find work in the Philips Physics Laboratory, 11 at the Bataafsche Petroleum Maatschappij.) In fundamental physics, Ornstein decided to contribute to the new physics of quantum mechanics, by measuring not just the wavelengths of spectral lines_-which many people were doing — but also the intensitiesfor which Utrecht was in a unique position. He therefore started calibrating photographic plates from different sources, testing their stability. He introduced the practice of illuminating with the light of a calibrated light source a part of each photographic plate which was used to obtain a flame spectrum, thus allowing accurate calibration of each individual plate. He used a stepped wedge, made by depositing platinum in six different thicknesses onto a $2 \mathrm{~cm}$ glass plate, to calibrate a large range of intensities at all colours simultaneously. He improved the stability of the thermocouple that served as the calibrating light source. In 1927 the Rockefeller Foundation donated a for those days very large Rowland grating: $13 \times 13 \mathrm{~cm}$, with 80000 lines and a radius of the Rowland circle of $R=5.65 \mathrm{~m}$.

Already before this, Ornsteins students Burger and Dorgelo (1924) had formulated summation rules linking quantum numbers of spectral lines, on the basis of relative line strengths (Table 1). These results forced the theoreticians to rethink the rules derived by Sommerfeld and Heisenberg (1922), which predicted intensities proportional to $j^{2}$, since the observations indicated a proportionality to $j(j+1)$. This was an important result as it showed that the transition probability depended on both initial and final state. This required a reformulation of the correspondence principle, although it was not obvious how, as witnessed by the following two points of view: 
Table 1 Conversion of experimentally determined relative intensities of calcium multiplet lines into generalized summation rules, after Burger and Dorgelo (1924)

\begin{tabular}{llllll}
\hline \multicolumn{6}{l}{ Measurements calcium multiplet (rounded) } \\
\hline$p_{1}-d_{3}$ & $p_{1}-d_{2}$ & $p_{1}-d_{1}$ & $p_{2}-d_{3}$ & $p_{2}-d_{2}$ & $p_{3}-d_{3}$ \\
\hline 1 & 18 & 100 & 19 & 54 & 25 \\
\hline \multicolumn{7}{c}{ Converted to tabular form } \\
\hline$J=2 j+1$ & $d_{1}(7 / 2)$ & $d_{2}(5 / 2)$ & $d_{3}(3 / 2)$ & Sum \\
\hline$p_{1}(5 / 2)$ & 100 & 18 & 1 & 119 \\
$p_{2}(3 / 2)$ & - & 54 & 19 & 73 \\
$p_{3}(1 / 2)$ & - & - & 25 & 25 \\
Sum & 100 & 72 & 45 & \\
\hline
\end{tabular}

\begin{tabular}{lllll}
\hline \multicolumn{6}{l}{ Generalized theory version } & & & \\
\hline $2 k+3$ & $2 k+1$ & $2 k-1$ & Sum \\
\hline $2 k+1$ & $12 k^{2}+12 k-9$ & $u$ & $12-u$ & $(2 k+1)(6 k+3)$ \\
$2 k-1$ & - & $12 k^{2}-3-u$ & $u$ & $(2 k-1)(6 k+3)$ \\
$2 k-3$ & - & - & $12 k^{2}-12 k-9$ & $(2 k-3)(6 k+3)$ \\
Sum & $(2 k+3)(6 k-3)$ & $(2 k+1)(6 k-3)$ & $(2 k-1)(6 k-3)$ & \\
\hline
\end{tabular}

The fundamental intensity-measurements of Ornstein etc. show that reality is much simpler and more arithmetical than expected from the Correspondence Principle.

(Sommerfeld, letter to Kramers 1924)

As regards the "intensities a la Ornstein" I fully share your view... Bohr and I have considered this carefully once more and concluded that the summation rules...follow unavoidably from the correspondence principle.

(Heisenberg, letter to Pauli 1924)

Note that the measurements of Ornstein were sufficiently known that Sommerfeld and Heisenberg did not need to specify them.

\section{The Utrecht Photometric Atlas of the Solar Spectrum}

Marcel Minnaert, one of the graduate students of Ornstein, utilized the accurate calibrations achieved by Ornstein and Moll for the interpretation of stellar spectra, and more particularly for lines in the solar spectrum obtained with the heliostat installed by Julius (Sect. 2.1). For the first time real intensities could be measured! In 1923 Minnaert conceived the concept of equivalent width (Minnaert 1927). In Munich, the graduate student Unsöld made a first attempt to link the line strengths of a stellar spectrum to abundances, and this led to the formulation of the curve of growth in 1929 by Minnaert and by Unsöld, who by then had moved to Kiel (Minnaert and van Assenbergh 1929; Unsöld et al. 1930). In 1931 Antonie Pannekoek, for whom the astronomy institute of the University of Amsterdam had been created, used Moll's microphotometer to publish the first curve of growth of a star, $\alpha$ Cygni (Pannekoek 1931). 

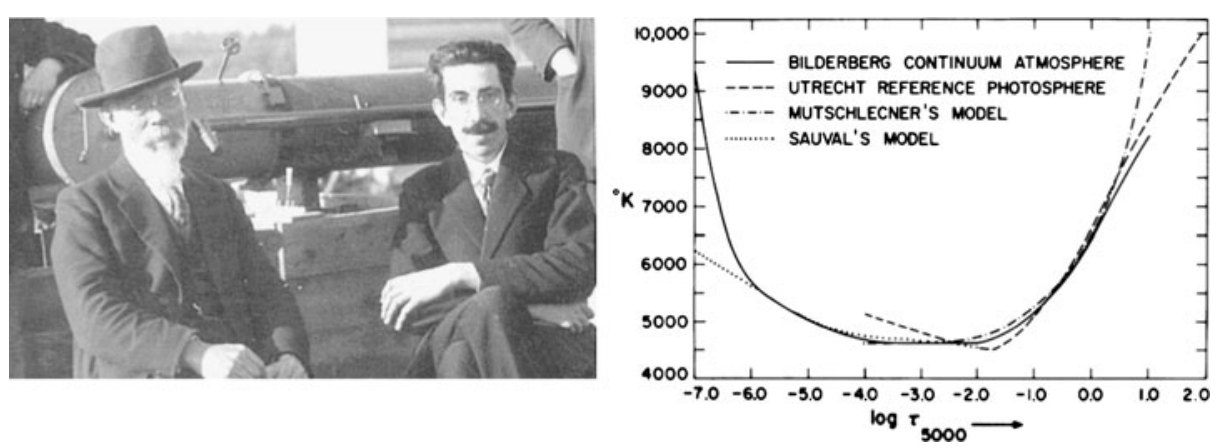

Fig. 2 Left: Anton Pannekoek and Marcel Minnaert. Right: Some early model atmospheres for the Sun

In Utrecht Minnaert embarked on a huge project: the Utrecht Photometric Atlas of the solar spectrum. The measurements for this were obtained on some hundred photographic plates by Gerard Mulder at the Mount Wilson Observatory in California in 1936, and in the following years Jaap Houtgast and Minnaert would take turns at Moll's microphotometer in measuring the spectral lines in the solar spectrum. The tracings of these measurements, automatically converted to a linear scale by a contraption invented by Houtgast, were published just before the Second World War: five copies left the Netherlands just before the Germans invaded and arrived safely in the USA (Minnaert et al. 1940). After the war, the enormous work of converting the line strengths into solar abundances was started; in the end the major contributor to this would be Charlotte Moore of the National Bureau of Standards in Washington. The result was published in 1966, thirty years after the observations were taken (!), as The Solar Spectrum (Moore et al. 1966).

Kees de Jager undertook the determination of the temperatures in the solar atmosphere as derived from the spectral lines. The resulting Utrecht Reference Photosphere of 1952 would remain a standard for years to come.

\section{X-Ray Spectroscopy}

Dutch industry and Dutch government were keen in the 1960s to participate in the new area of space research. The Royal Netherlands Academy of Arts and Sciences, in collaboration with several universities set up working groups from which the Space Research Organisation of the Netherlands (SRON) would eventually emerge in 1983. In 1961 Kees de Jager started the working group Space Research of Sun and Stars at Utrecht University, and given his background it is not surprising that he chose X-ray spectroscopy as his field of action.

It is perhaps surprising that the scientist hired in 1970 to develop theoretical X-ray spectroscopy in the Utrecht working group, was trained not in the Utrecht Minnaert school but in Amsterdam, at the prestigious Zeeman Laboratory. This scientist was Rolf Mewe. Mewe would devote his career to work on the code to compute the X-ray spectrum of a hot plasma, always extending and improving its scope, keeping track with the improved physical understanding of the processes involved and with the increased capabilities of new generations of computers (see Kaastra and Mewe 2005).

In preparing subsequent incarnations of the code Mewe was helped by a number of scientists, in particular by Jelle Kaastra and Duane Liedahl, hence the name under which the code is now known: MeKaL (Mewe et al. 1995). 

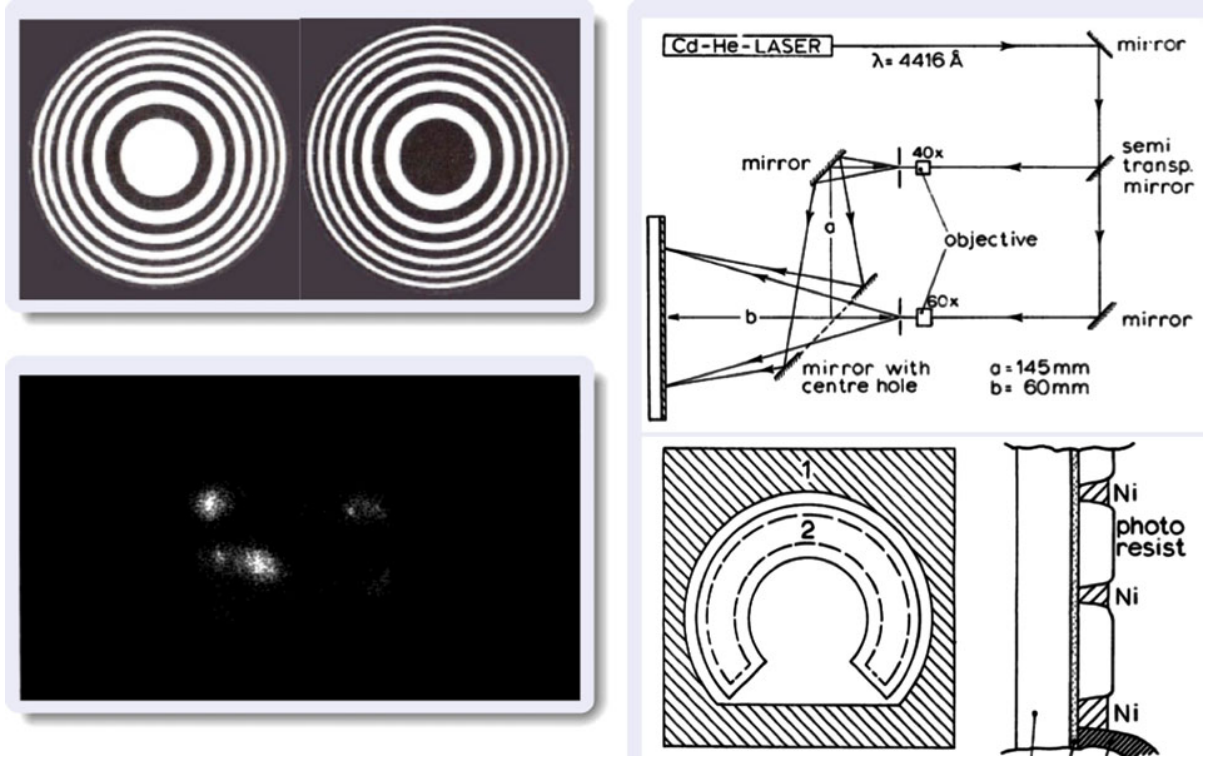

Fig. 3 Left above: two versions of Fresnel zone plates, with central zone open or closed. Left below: the Sun at $51 \AA$ (Si X) on October 3, 1967. Right: Fresnel zone plate production (for explanation see text; from Dijkstra et al. 1971)

\subsection{From Image of the Sun to Spectra of the Stars}

The first method used in Utrecht to image the Sun in X-rays used Fresnel zone plates. These consist of a large number of alternately transparent and opaque rings (see Fig. 3), and provide monochromatic images. The first Fresnel plates were made by Le Poole in Delft, with electron-optical imaging. This limited the radius of the plate to $r_{N} \simeq 1 \mathrm{~mm}$. Together with the wavelength of e.g. $\lambda=5 \mathrm{~nm}$ and a feasible focal length $f \simeq 0.4 \mathrm{~m}$, this required rings of widths $\Delta r_{N} \simeq 1 \mu \mathrm{m}$-from the relation $2 r_{N} \Delta r_{N}=f \lambda$.

To limit the intensity of the zero-order image, plates with opaque central regions are preferred, requiring large-diameter plates, and for this reason a new technique was developed by Dijkstra et al. (1971). First a zone plate image was produced as an interferogram from a beam-split $\mathrm{Cd}-\mathrm{He}$ laser, this image was contact printed onto chromium substrate, and nickel was deposited in the exposed areas (Fig. 3). Finally, the Fresnel zone plates were strengthened and lifted from the substrate. Four Fresnel plates, for four wavelengths, were flown with Aerobee rockets in 1967 and gave one useful image of the Sun, in a Si X line at $51 \AA$.

The technique developed for the solar X-ray images enabled the Utrecht working group, and later SRON to become the Lead Investigator for the grating spectrographs of the Einstein and EXOSAT satellites, launched in November 1978 and May 1983, respectively. The Objective Grating Spectrometer of Einstein (OGS, Dijkstra et al. 1978) covered the soft X-ray domain up to wavelengths of tens of Ångstroms. The Transmission Grating Spectrometer of EXOSAT (TGS, Brinkman et al. 1980) extended much further into the XUV domain, to wavelengths of several hundred Ångstrom, including the He II Ly $\alpha$ edge and line at $304 \AA$. Both the OGS and the TGS had to be moved into position, and the fear that it would get stuck and not be removable again limited the number of observations scheduled 

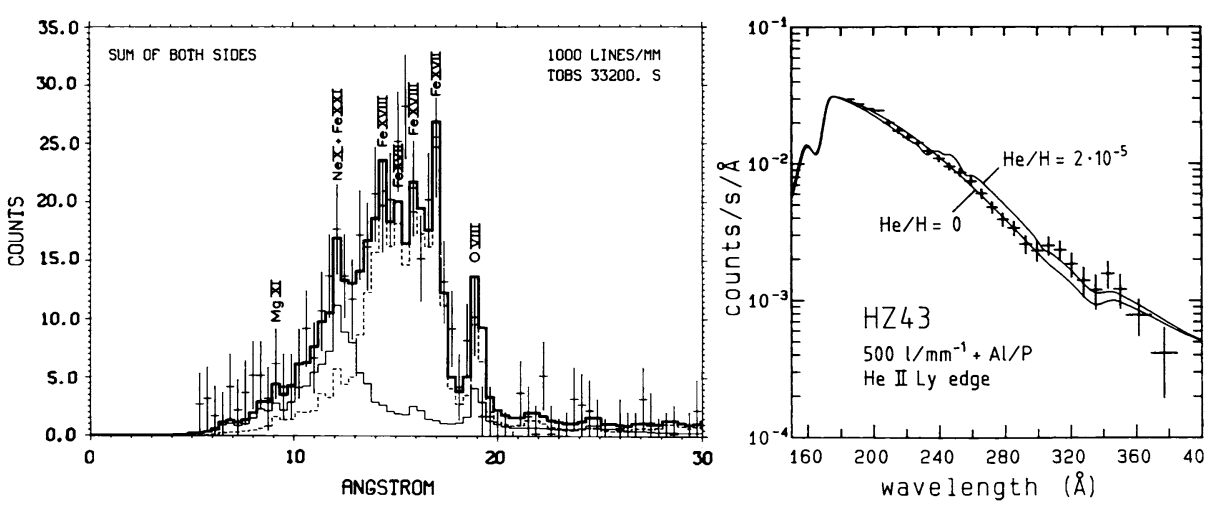

Fig. 4 Left: Einstein OGS spectrum of Capella. Lines of a 5 million degree plasma (dashed line) are combined with those of a 10 million degree plasma (thin solid line; from Mewe et al. 1982). Right: EXOSAT TGS spectrum of the white dwarf HZ 43 provides a strong upper limit to the amount of helium in the atmosphere (from Heise et al. 1988)

with these instruments. Nonetheless, the grating produced first real spectra of a varied list of targets, and important results were obtained in particular on the X-ray emission of solartype stars and of white dwarfs (Fig. 4). The X-ray spectrum of Capella taken with the OGS showed coronal emission of a plasma with at least two different temperatures. From the TGS observation obtained later it could be shown that the distribution of emission measures $\left(\mathrm{EM} \equiv \int n_{e}^{2} d V\right)$ is not monotonous, but shows maxima near 3 and 18 million degrees and a minimum in between (Lemen et al. 1989). The X-ray spectrum taken with the TGS of the hot white dwarf HZ 43 enabled the determination of a very strong upper limit to the helium abundance in its atmosphere, in combination with a much more accurate determination of the interstellar absorption towards this object.

\subsection{Spectrographs on Chandra and on XMM-Newton}

Since Spectroscopy is the queen of astronomy, it is clear that any major X-ray observatory should have a spectrograph. The expertise of SRON, obtained with the Einstein and EXOSAT observatories, enabled it to become the principal investigator for the Low-energy Transmission Grating System on Chandra as well as for the Reflection Grating Spectrograph on $X M M$-Newton. The production of these large spectrographs requires extensive international collaboration, and thus both spectrographs are the result of international team effort. To allow full coverage of 1-200 A Chandra also houses a High-energy Transmission Grating Spectrograph, for which MIT was selected as the principal investigator. These observatories were launched in July 1999 and December 1999, respectively.

Chandra LETGS This instrument was designed and built by SRON and MPE Garching (Brinkman et al. 2000). It comprises grating facets of gold with 1000 lines $/ \mathrm{mm}$, supported with a pitch of $25 \mu \mathrm{m} / 2 \mathrm{~mm}$. The gratings are placed immediately behind the X-ray mirror system (see Fig. 5). The (mainly) iron complex between 12 and $20 \AA$ is fully resolved with the LETGS spectrograph. The spectra of Capella and Procyon provide accurate temperatures, from ratios of different ions of the same element, and densities, from the ratio of the resonance, intercombination and forbidden lines in helium-like triplets (Fig. 5). The temperatures and densities are comparable to those of the plasma responsible for the X-ray 

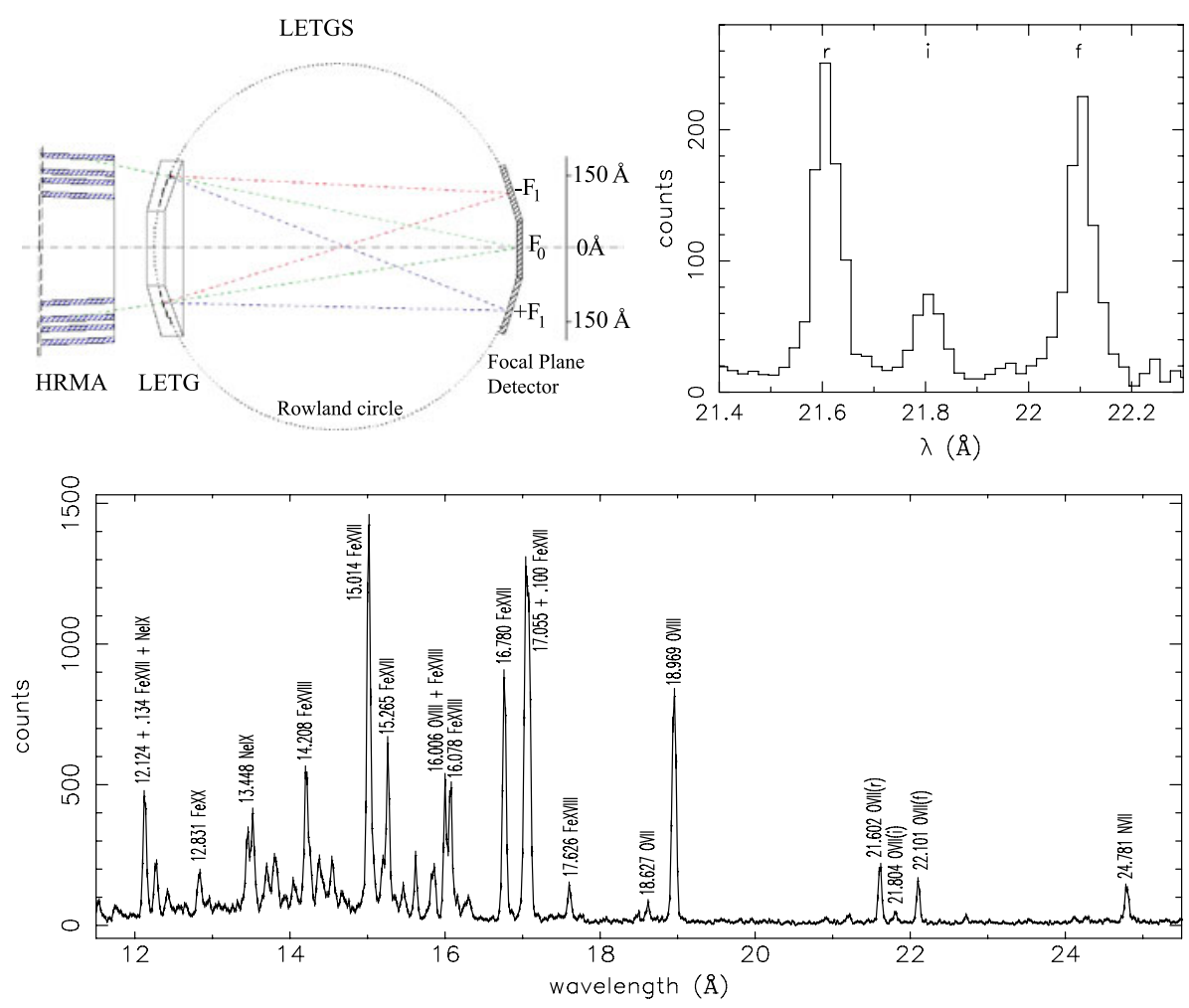

Fig. 5 Above left: schematic view of the Chandra LETGS with the mirrors (left) and CCDs (right). Below: LETGS spectrum of Capella, from Mewe et al. (2001). Note that the range shown corresponds to an unresolved single maximum in Fig. 4. Above right: The O VII triplet in the LETGS spectrum of Procyon, after Ness et al. (2001)

emission of the Sun. The quality of the observations is now such that the observed spectra can be used to calibrate theoretical line computations.

$X M M$-Newton RGS The grazing incidence Reflection Grating Assemblies for the RGS were built by the University of California at Berkeley and the Lawrence Livermore National Laboratory (den Herder et al. 2001), and are located behind two of the three mirror assemblies (Fig. 6). It combines high spectral resolution with high throughput in the range 5-35 A. Moreover, the intrinsic high wavelength dispersion of the RGS enables high-resolution spectroscopy of extended objects, like supernova remnants, up to diameters of a few arcminutes.

The optical configuration requires a slightly variable line density; the average line density is 645 grooves $/ \mathrm{mm}$. For the detector special chips were developed by GEC-EEV (UK) with low read-out noise $\left(<5 \mathrm{e}^{-}\right)$and hardened against $\mathrm{MeV}$ proton radiation. The camera system was integrated, tested and calibrated by SRON. One of the first results of the RGS was the surprising absence of the lines of the cooled gas in several cooling flows in clusters of galaxies: apparently the gas is prevented from cooling below a lower threshold (Fig. 6). Another field which is opened up by the high resolution X-ray spectra of both LETGS and RGS is that of the non-equilibrium plasmas in supernova remnants, of which an example is shown in Fig. 7. 


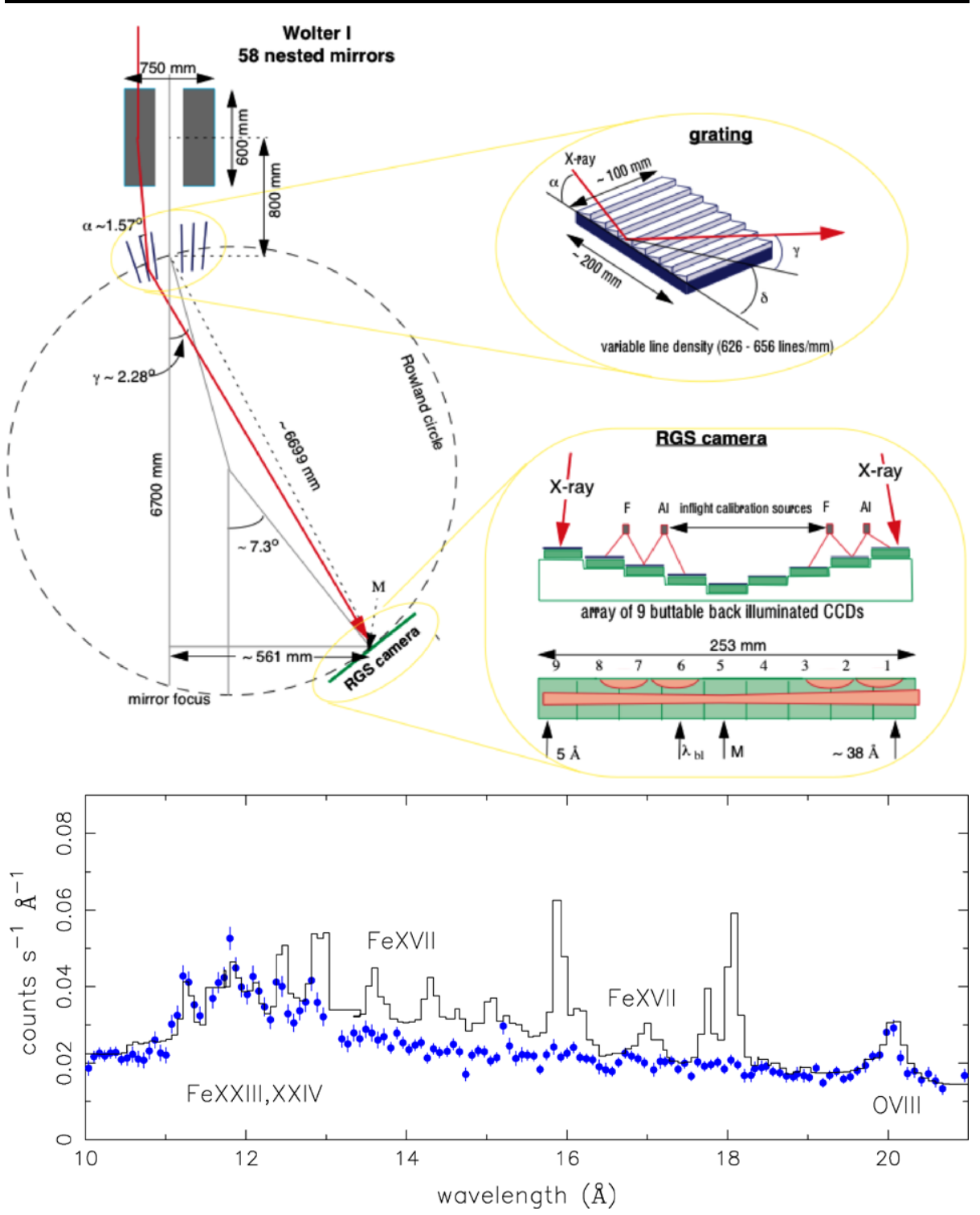

Fig. 6 Above: schematic view of the XMM RGS with the mirrors (above), gratings and CCDs (below right); after Brinkman et al. (1998). Below: the RGS spectrum of the cluster of galaxies Sersic 159-03 does not show the expected lines of the coolest part of the cooling flow; after de Plaa et al. (2006), see also Peterson et al. (2001)

\section{Summary}

We see that a direct line connects the first modern experimental physics in Utrecht at the end of the 19th century, to the current effort at SRON in high-resolution X-ray spectroscopy in the beginning of the 21th century. From the beginnings of the research on heat radiation by Buys Ballot and Julius, an extensive experimental study was set up by Ornstein in op- 


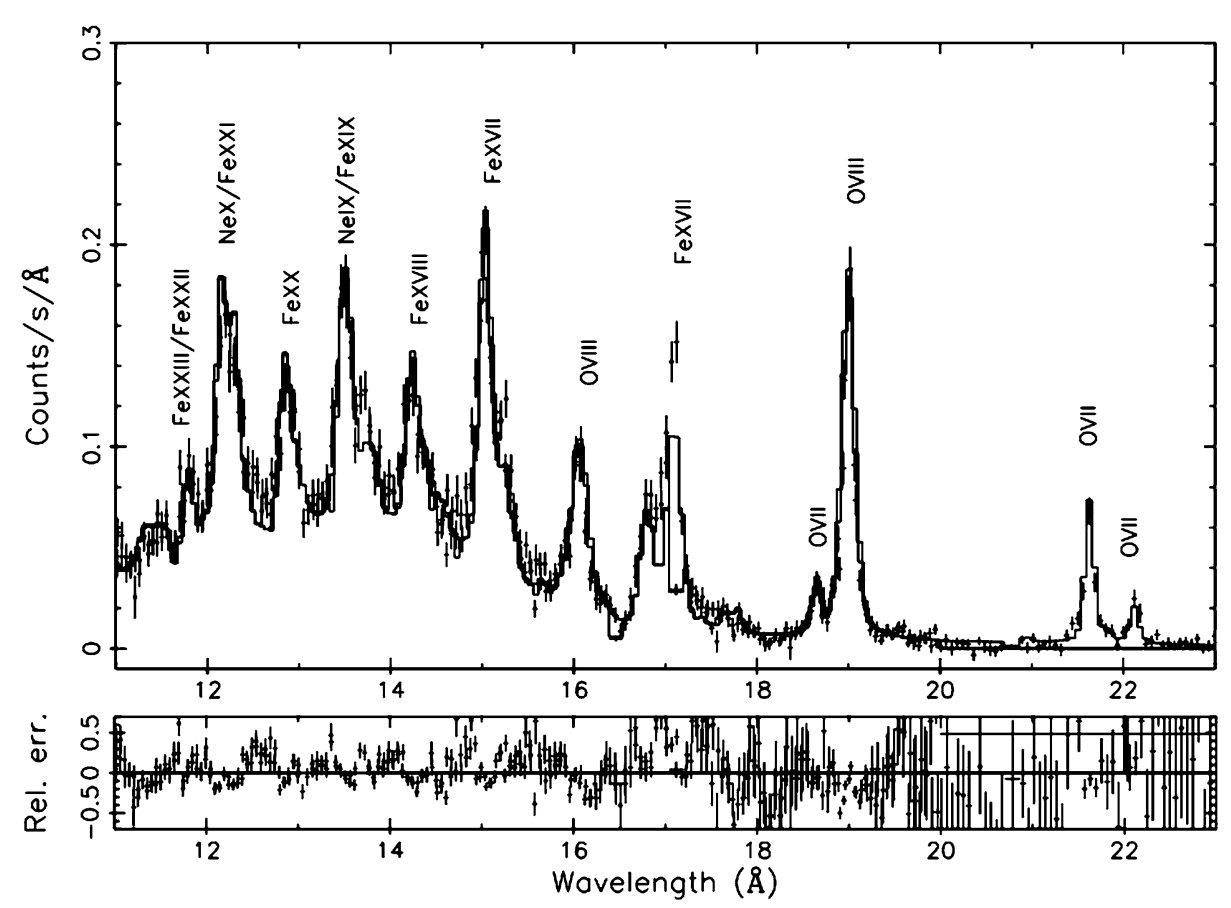

Fig. 7 RGS spectrum of the supernova remnant N103B; from van der Heyden et al. (2002). This spectrum illustrates the capability of the RGS to produce high-resolution spectra from extended objects

tical spectroscopy, with emphasis on careful calibration of intensities. His techniques were transported to astronomical measurements by Minnaert, whose student De Jager extended the spectroscopy into the ultraviolet and X-rays at the newly founded institute for space research. Techniques initially developed for imaging the Sun in X-rays turned out to be suitable for the production of X-ray gratings as well, and thus SRON became a principal player in the development of the spectrographs on Einstein, EXOSAT, Chandra and XMM-Newton.

Open Access This article is distributed under the terms of the Creative Commons Attribution Noncommercial License which permits any noncommercial use, distribution, and reproduction in any medium, provided the original author(s) and source are credited.

\section{References}

A.C. Brinkman, J.H. Dijkstra, W. Geerlings, F.A. van Rooijen, C. Timmerman, P.A.J. de Korte, Appl. Opt. 19, 1601 (1980)

A.C. Brinkman et al., in Science with XMM, ESTEC Noordwijk, published online at http://xmm.esac.esa.int/ external/xmm_science/workshops/1st_workshop (1998)

A.C. Brinkman, Th. Gunsing, J.S. Kaastra et al., SPIE 4012, 81 (2000)

H.C. Burger, H.B. Dorgelo, Z. Phys. 23, 258 (1924)

J.W. den Herder, A.C. Brinkman, S.M. Kahn et al., Astron. Astrophys. 365, L7 (2001)

J. de Plaa, N. Werner, A.M. Bykov, J.S. Kaastra, M. Mendez, J. Vink, J.A.M. Bleeker, M. Bonamente, J.R. Peterson, Astron. Astrophys. 452, 397 (2006)

J.H. Dijkstra, W. de Graaff, L.J. Lantwaard, in New Techniques in Space Astronomy, ed. by F. Labuhn, R. Lüst. IAU Symposium, vol. 41 (1971), p. 207 
J.H. Dijkstra, L.J. Lantwaard, C. Timmerman, in New Instrumentation for Space Astronomy, ed. by K.A. van der Hucht, G.S. Vaiana (1978), p. 257

J. Heise, F.B.S. Paerels, J.A.M. Bleeker, A.C. Brinkman, Astrophys. J. 334, 958 (1988)

H.G. Heijmans, Wetenschap Tussen Universiteit en Industrie. De Experimentele Natuurkunde in Utrecht onder W.H. Julius en L.S. Ornstein 1896-1940 (Erasmus Publishing, Rotterdam, 1994)

J.S. Kaastra, R. Mewe, in X-ray Diagnostics of Astrophysical Plasmas, ed. by R.S. Smith. American Institute of Physics, CP, vol. 774 (2005), p. 1

J.R. Lemen, R. Mewe, C.J. Schrijver, A. Fludra, Astrophys. J. 341, 474 (1989)

R. Mewe, E.H.B.M. Gronenschild, N.J. Westergaard, J. Heise, F.D. Seward, T. Chlebowski, N.P.M. Kuin, A.C. Brinkman, J.H. Dijkstra, H.W. Schnopper, Astrophys. J. 260, 233 (1982)

R. Mewe, J.S. Kaastra, D.A. Liedahl, Legacy 6, 16 (1995)

R. Mewe, A.J.J. Raassen, J.J. Drake, J.S. Kaastra, R.L.J. van der Meer, D. Porquet, Astron. Astrophys. 368, 888 (2001)

M. Minnaert, Z. Phys. 45 (1927)

M. Minnaert, B. van Assenbergh, Z. Phys. 53 (1929)

M. Minnaert, J. Houtgast, G.F.W. Mulders, Photometric Atlas of the Solar Spectrum From $\lambda 3612$ to $\lambda 8771$, with an appendix from $\lambda 3332$ to $\lambda 3637$ (Utrecht Sterrewacht Sonnenborgh, 1940)

L. Molenaar, De Rok van het Universum. Marcel Minnaert Astrofysicus 1893-1970 (Uitgeverij Balans, Amsterdam, 2003)

W.J.H. Moll, Proc. Phys. Soc. 33, 207 (1921)

W.J.H. Moll, Phys./Ned. Tijdschr. Nat. 6, 233 (1926)

Ch.E. Moore, M. Minnaert, J. Houtgast, The Solar Spectrum From $2935 \AA$ to $8770 \AA$ (National Bureau of Standards, Washington, 1966)

J.-U. Ness, R. Mewe, J.H.M.M. Schmitt, A.J.J. Raassen, D. Porquet, J.S. Kaastra, R.L.J. van der Meer, V. Burwitz, P. Predehl, Astrophys. J. 367, 282 (2001)

A. Pannekoek, Proc. Acad. Sci. Amst. 34, 755 (1931)

J.R. Peterson, F.B.S. Paerels, J.S. Kaastra, M. Arnaud, T.H. Reiprich, A.C. Fabian, R.F. Mushotzky, J.G. Jernigan, I. Sakelliou, Astron. Astrophys. 365, L104 (2001)

A. Sommerfeld, W. Heisenberg, Z. Phys. 11, 131 (1922)

A. Unsöld, O. Struve, C.T. Elvey, Z. Astrophys. 1, 314 (1930)

P.H. van Cittert, Phys./Ned. Tijdschr. Nat. 6, 224 (1926)

K.J. van der Heyden, E. Behar, J. Vink, A.P. Rasmussen, J.S. Kaastra, J.A.M. Bleeker, S.M. Kahn, R. Mewe, Astron. Astrophys. 392, 955 (2002)

B. Willink, De Tweede Gouden Eeuw. Nederland en de Nobelprijzen voor Natuurwetenschappen 1870-1940 (Bert Bakker, Amsterdam, 1998) 